\title{
Autophagy protects bone marrow mesenchymal stem cells from palmitate-induced apoptosis through the ROS-JNK/p38 MAPK signaling pathways
}

\author{
YONGYI LIU ${ }^{1}$, NING WANG ${ }^{1}$, SHAOKUN ZHANG $^{2}$ and QINGWEI LIANG $^{3}$ \\ ${ }^{1}$ Department of Orthopedics, The First Hospital of China Medical University, Shenyang, Liaoning 110000; \\ ${ }^{2}$ Department of Orthopedics, The First Affiliated Hospital of Zhengzhou University, Zhengzhou, Henan 450052; \\ ${ }^{3}$ Department of Sports Medicine, The First Hospital of China Medical University, Shenyang, Liaoning 110000, P.R. China
}

Received January 9, 2018; Accepted May 18, 2018

DOI: $10.3892 / \mathrm{mmr} .2018 .9100$

\begin{abstract}
In recent years, the association between saturated fatty acids (FA) and bone cells has received a high level of attention. Previous studies have shown that palmitate (PA), a common saturated FA, can cause apoptosis in bone marrow mesenchymal stem cells (BMSCs). However, whether PA can induce autophagy, an important intracellular protection mechanism that is closely associated with apoptosis, in BMSCs is still unknown; the association between autophagy and apoptosis is also unclear. The aim of the present study was to determine whether PA can induce autophagy in BMSCs. When BMSCs were treated with PA for $>18 \mathrm{~h}, \mathrm{p} 62$ began to accumulate, indicating that autophagic flux was impaired by prolonged exposure to PA. In addition, the proportion of apoptotic cells was increased when autophagy was inhibited by the autophagy inhibitor 3-methyladenine. Furthermore, inducing autophagy by pretreating cells with rapamycin, a known inducer of autophagy, markedly reduced PA-induced apoptosis, suggesting that autophagy may serve a protective role in PA-induced apoptosis in BMSCs. PA also increased intracellular reactive oxygen species (ROS) production, which was decreased by the antioxidant N-Acetyl-cysteine, and promoted the activation of c-Jun N-terminal kinases (JNKs) and $\mathrm{p} 38$ mitogen-activated protein kinase (MAPK). The addition of JNK and p38 MAPK inhibitors substantially reduced autophagy. Therefore, the results indicated that PA can induce autophagy in BMSCs and protect cells from PA-induced apoptosis through the ROS-JNK/p38 MAPK signaling pathways. These results may improve the general understanding of the mechanisms through which BMSCs adapt to PA-induced
\end{abstract}

Correspondence to: Professor Qingwei Liang, Department of Sports Medicine, The First Hospital of China Medical University, 155 North Nanjing Street, Shenyang, Liaoning 110000, P.R. China E-mail: cmulqw@sina.com

Key words: autophagy, bone marrow mesenchymal stem cells, palmitate, protector, reactive oxygen species apoptosis. The present study also provides a novel approach for the prevention and treatment of PA-induced lipotoxicity.

\section{Introduction}

Osteoporosis is an important public health issue, especially for postmenopausal women and the elderly, and is caused by unequilibrated bone remodeling resulting from decreased bone formation and/or accelerated bone resorption (1). In osteoporotic patients, bone marrow fat increases and bone mass decreases (2). Previous studies have confirmed that increased bone marrow fat may have toxic effects on bone cells through the release of saturated fatty acids (FA), which results in reduced bone mass (3-5). Moreover, high saturated FA consumption was shown to be associated with an increased risk of osteoporotic fractures (6). As the most common saturated FA in humans, palmitate (PA) has been widely investigated to evaluate the effects of FAs on various cell types $(7,8)$. In bone cells, lipotoxicity has been reported in osteoblasts after exposure to PA $(5,9)$.

Autophagy is an evolutionarily conserved process for the catabolism of damaged proteins and organelles in the cell to maintain intracellular environmental homeostasis by 'self-clearing' $(10,11)$. Studies suggest that moderate autophagy is required for coping with cellular stress, inhibition of apoptosis, and is conducive to cell survival; however, the persistence of adverse factors can lead to excessive autophagy, which induces cell death (12). Autophagy can be found in all types of bone cells, and plays an important role in osteogenesis $(13,14)$. Bone marrow mesenchymal stem cells (BMSCs) are multipotent cells that can differentiate into osteoblasts and other cell types such as adipocytes and chondrocytes (15). Previous studies have shown that PA can cause apoptosis in human MSCs $(15,16)$; however, it is still unknown whether PA can cause autophagy, and the effects of autophagy on PA-induced apoptosis remain unclear.

Previous studies have confirmed that PA released by adipocytes can induce apoptosis in osteoblasts by increasing reactive oxygen species (ROS) production (17). In addition, ROS has been shown to be a strong signal for the activation of c-Jun N-terminal kinases (JNKs); JNK activation may mediate 
antioxidant responses including the induction of autophagy and cell death (18). Furthermore, the p38 mitogen-activated protein kinase (MAPK) has been shown to regulate autophagy in response to chemotherapy drugs (19). However, whether JNK and p38 MAPK play a role in PA-induced autophagy in BMSCs remains unclear. Therefore, we studied the correlation of ROS, JNK, and p38 MAPK with autophagy induced by PA, as well as the role of autophagy in apoptosis in BMSCs.

\section{Materials and methods}

Reagents. Antibodies against cleaved caspase-3 (9679S, 1:800), p62 (39749S, 1:800), JNK (9252S, 1:1,000), phospho-JNK (4668S, 1:800), p38MAPK (8690S, 1:1,000), phospho-p38 MAPK (4511S, 1:800), $\beta$-Actin $(8457 \mathrm{~S}, 1: 3,000)$ and GAPDH (5174S, 1:3,000) were purchased from Cell Signaling Technology, Inc. (Danvers, MA, USA). Antibodies for LC3B-II were purchased from Abcam (ab51520, 1:3,000; Cambridge, MA, USA). Fetal bovine serum (FBS) was obtained from Gemini Bio Products (900108; Woodland, CA, USA). FA-free bovine serum albumin (BSA) was obtained from Solarbio (A8850; Beijing, China). PA (P0500), SP600125 (S5567), and SB203580 (S8307) were purchased from Sigma-Aldrich (Merck KGaA, Darmstadt, Germany). Rapamycin (RA, S1039) and 3-methyladenine (3-MA, S2767) were obtained from Selleck Chemicals (Houston, TX, USA).

Cell culture. BMSCs derived from Sprague-Dawley rats were purchased from Cyagen Biosciences (RASMX-90011; Shanghai, China). According to tests conducted by the supplier, the majority of BMSCs were positive for CD29, CD44 and CD90; negative for CD11, CD34, and CD45; and had the potential to differentiate into osteoblasts, adipocytes, and chondrocytes. Cells were plated in growth media at $37^{\circ} \mathrm{C}$ with $5 \% \mathrm{CO}_{2}$. Growth media was composed of DMEM/F12 (SH30026.01B; HyClone; GE Healthcare Life Sciences, Logan, UT, USA) and 10\% FBS. After reaching approximately $90 \%$ confluence, cells were trypsinized, detached, and passaged. BMSCs were between passages 3 and 8 for all experiments.

PA preparation. The PA stock solution was prepared, and treatment was performed as previously described $(5,9)$. Briefly, the stock solution was prepared by dissolving PA (final concentration: $10 \mathrm{mM}$ ) in $0.01 \mathrm{M} \mathrm{NaOH}$ at $70^{\circ} \mathrm{C}$ for $30 \mathrm{~min}$ with FA-free BSA. The molar ratio of PA to BSA was 7:1. The stock solution was added to a serum-free medium to achieve a final concentration of $0.5 \mathrm{mM}$ PA and $1 \%$ BSA. Control cells were treated with $1 \%$ BSA only.

Cell viability assay. BMSCs were seeded in 96-well plates (6x10 3 cells/well) for $24 \mathrm{~h}$. When $80 \%$ confluence was achieved, the culture medium was replaced with medium with or without increasing concentrations of PA (0.125-0.50 mM). Separately, BMSCs were also treated with $0.5 \mathrm{mM}$ PA for different durations $(0,3,6,12,18$ and $24 \mathrm{~h})$. Following PA treatment, $20 \mu \mathrm{l}$ 3-[4,5-dimethylthiazol-2-y]-2,5-diphenyltetrazolium bromide (MTT, KGA311; KeyGEN, Nanjing, China) was added to each well and incubated for $4 \mathrm{~h}$ at $37^{\circ} \mathrm{C}$. Thereafter, the medium containing MTT was discarded and $150 \mu$ l dimethyl sulfoxide (DMSO, ST038; Beyotime, Nantong, China) was added to each well and incubated for $10 \mathrm{~min}$ in the dark at room temperature. The absorbance at $490 \mathrm{~nm}$ (A490) was measured using a microplate reader. The cell survival rate (\%) was calculated as follows: Experimental group A490/control group A490.

Apoptosis analysis. BMSCs were seeded in 6-well plates ( $2 \times 10^{5}$ cells/well) and treated with PA $(0.125-0.50 \mathrm{mM})$ for $24 \mathrm{~h}$. Separately, the BMSCs were also treated with $0.5 \mathrm{mM}$ PA for $24 \mathrm{~h}$ with or without 3-methyladenine (3-MA; $5 \mathrm{mM}$ ) or rapamycin (RA; $5 \mu \mathrm{M})$ pretreatment. Cells were then evaluated using the Annexin V-FITC/PI apoptosis detection kit according to the manufacturer's instructions (AD10; Dojindo Molecular Technologies, Inc., Kumamoto, Japan). Briefly, BMSCs were harvested and washed three times with PBS before being resuspended in $400 \mu \mathrm{l}$ binding buffer, then $5 \mu \mathrm{l}$ Annexin V-FITC and PI were added to the samples and incubated for $15 \mathrm{~min}$ in the dark at room temperature. The samples were analyzed by a flow cytometer equipped with Modfit LT 3.0 (BD Biosciences, Franklin Lakes, NJ, USA), and results were analyzed using Cell Quest software. Different subpopulation was distinguished using the following criteria: Q1, necrotic cells (FITC-/PI+); Q2, late apoptotic cells (FITC+/PI+); Q3, viable cells (TITC-/PI-); Q4, early apoptotic cells (FITC+/PI-). The apoptotic rate was determined as the percentage of $\mathrm{Q} 2+\mathrm{Q} 4$.

Measurement of caspase-3/9 activity. The detection of caspase- 3 and caspase- 9 activity was performed using caspase colorimetric assay kits according to the manufacturer's instructions (KGA202 and KGA402; Keygen Biotech, Nanjing, China). Briefly, BMSCs were harvested and washed twice with PBS after treatment for $24 \mathrm{~h}$, and then resuspended in lysis buffer and incubated on ice for $1 \mathrm{~h}$. Subsequently, lysates were centrifuged at $10,000 \mathrm{x}$ g for $1 \mathrm{~min}$ and the concentration of each sample was detected using a bicinchoninic acid (BCA) protein assay kit (AR1110; Boster, Wuhan, China). Accordingly, $150 \mu \mathrm{g}$ total protein from each sample was combined with PBS to achieve a final volume of $50 \mu 1$, then the samples were incubated with $5 \mu$ caspase substrate in the dark at $37^{\circ} \mathrm{C}$ for $4 \mathrm{~h}$. The A405 was measured using a microplate reader. Caspase- 3 and caspase- 9 activity were calculated as follows: Experimental group A405/control group A405.

Detection of intracellular ROS. BMSC ROS levels were detected using the ROS detection kit (S0033; Beyotime). Cells ( $2 \times 10^{5}$ cells/well) in 6 -well plates were treated with variable concentrations of PA for $6 \mathrm{~h}$ at $37^{\circ} \mathrm{C}$. Thereafter, BMSCs were incubated with DCFH-DA $(5 \mathrm{mM})$ at $37^{\circ} \mathrm{C}$ for $20 \mathrm{~min}$, and washed three times with serum-free medium. ROS levels were measured using an inverted fluorescence microscope (IX71; Olympus Corporation, Tokyo, Japan). In addition, cells were harvested and washed twice with serum-free medium after treatment as indicated for $6 \mathrm{~h}$ at $37^{\circ} \mathrm{C}$, then resuspended in $500 \mu 1$ PBS with $5 \mathrm{mM}$ DCFH-DA; the samples were then incubated for $20 \mathrm{~min}$ in the dark at $37^{\circ} \mathrm{C}$. Intracellular ROS was detected by flow cytometry (BD Biosciences) and analyzed using Cell Quest software.

Western blotting. After treatment, BMSCs were extracted with lysis buffer for $30 \mathrm{~min}$ and centrifuged at $14,000 \mathrm{x} \mathrm{g}$ for $30 \mathrm{~min}$ 
at $4^{\circ} \mathrm{C}$. The supernatant containing total protein was harvested. The protein concentration in the extract was determined using the BCA protein assay (AR1110; Boster). Equal amounts of protein were loaded into each lane, separated by a 10 or 12\% SDS-PAGE, and electroblotted onto PVDF membranes (LC2002; EMD Millipore, Billerica, MA, USA) at 50 or $70 \mathrm{~V}$ for $100 \mathrm{~min}$ at $4^{\circ} \mathrm{C}$. The membrane was then blocked in $5 \%$ non-fat milk for $1 \mathrm{~h}$ at room temperature. Subsequently, proteins were incubated using primary antibodies at 1:500 or 1:1,000 dilution overnight at $4^{\circ} \mathrm{C}$. Next, the membrane was incubated with goat anti-rabbit secondary antibody conjugated to horseradish peroxidase (ZB-2306, 1:8,000; ZSGB-BIO, Beijing, China) for $2 \mathrm{~h}$ at room temperature. The protein signal was enhanced using a chemiluminescence system (DNR MF-ChemiBIS 3.2) and the band density was measured using ImageJ.

Transmission electron microscopy. After treatment with or without PA $(0.5 \mathrm{mM})$ for $6 \mathrm{~h}$ at $37^{\circ} \mathrm{C}$, the cells were collected and fixed with $5 \%(\mathrm{v} / \mathrm{v})$ glutaraldehyde. The cells were then conventionally dehydrated, embedded, sectioned, and stained. Transmission electron microscopy (TEM, H-7650; Hitachi, Ltd., Tokyo, Japan) was used to observe the formation of autophagosomes. The number of intracellular autophagosomes was counted in every ten fields.

Immunofluorescence staining. Cells $\left(8 \times 10^{3}\right.$ cells/well) were seeded in 24-well plates and treated with PA $(0.50 \mathrm{mM})$ for $6 \mathrm{~h}$ with or without 3-MA $(5 \mathrm{mM})$ or N-Acetyl-cysteine (NAC; $1 \mathrm{mM}$, S0077; Beyotime). Subsequently, cells were fixed in 4\% paraformaldehyde (P0099; Beyotime) for $15 \mathrm{~min}$ at room temperature and washed twice with PBS, then permeabilized with $0.2 \%$ triton X-100 (P0096; Beyotime) for $5 \mathrm{~min}$ at room temperature. Thereafter, cells were washed with PBS, incubated with 5\% BSA for $1 \mathrm{~h}$, and incubated in anti-LC3 antibody (ab51520, 1:200; Abcam) overnight at $4^{\circ} \mathrm{C}$. The cells were then incubated with goat anti-rabbit secondary antibody labeled with fluorescein (ZF-0511, 1:400; ZSGB-BIO) for $1 \mathrm{~h}$ at room temperature before staining with DAPI (C1006; Beyotime). Samples were photographed using a wide field fluorescent microscope (IX71; Olympus Corporation). The number of LC3 positive cells in each microscopic field was divided by the number of nuclei in the same field, and was regarded as the rate of LC3 positive cells.

Statistical analysis. All experiments were repeated three times. Quantitative data are presented as the mean \pm standard error mean. SPSS 17.0 software (SPSS, Inc., Chicago, IL, USA) was used for analysis. Statistical analysis was performed using Student's t-test or one-way analysis of variance followed by a Bonferroni post hoc test for multiple comparisons. Differences between groups were considered statistically significant at $\mathrm{P}<0.05$.

\section{Results}

PA induces apoptosis in BMSCs. Considering that PA is the most ubiquitous FA in humans (including their bone marrow), and since PA induces apoptosis in other systems, in this study we tested whether it also induces these processes in
BMSCs (20-22). Here we used a dose of PA that corresponds to the concentration secreted by adipocytes into the media (5), was already used in previous studies looking at PA-induced lipotoxicity in bone cells $(5,9)$, and closely corresponds to the levels of PA found in bone marrow of human subjects (23).

To study the toxic effects of PA on BMSCs, cells were treated with increasing concentrations of PA $(0.125-0.50 \mathrm{mM})$. PA caused a marked reduction of cell viability in BMSCs in a time- and dose-dependent manner (Fig. 1A). In addition, we measured the levels of cleaved caspase- 3 in BMSCs. As shown in Fig. 1B, PA triggered the cleavage of caspase-3 in a dose-dependent manner. Moreover, flow cytometry revealed a marked increase in apoptosis in BMSCs treated with PA (Fig. 1C and D). Collectively, these results indicated that PA can induce apoptosis in BMSCs.

PA induced autophagy in BMSCs, but autophagic flux decreased over time. To determine whether PA stimulated autophagy in BMSCs, we used TEM to observe and count autolysosomes. As shown in Fig. 2A and B, after treatment with PA $(0.50 \mathrm{mM})$ for $6 \mathrm{~h}$, the number of autolysosomes in BMSCs increased remarkably compared to control groups. In addition, fluorescence microscopy was used to observe cells with punctate aggregation (autolysosomes) of internal LC3 (Fig. 2C). Punctate aggregation increased substantially after treatment with PA for $6 \mathrm{~h}$ compared to the control groups (Fig. 2D); this result was consistent with the findings obtained via TEM. When cells were treated with 3-MA, a classical inhibitor of autophagy, punctate aggregation greatly decreased compared to the PA-treated groups without 3-MA (Fig. 2C).

Autophagic flux decreased after longer exposure to PA. We also measured the levels of LC3 and p62. After BMSCs were treated for $6 \mathrm{~h}$ with increasing concentrations of PA (0.125-0.50 mM), the LC3-II levels increased considerably, while p62 levels decreased (Fig. 3A and B) in a dose-dependent manner. Furthermore, when BMSCs were treated with PA $(0.5 \mathrm{mM})$ at various time-points, LC3B-II levels increased gradually starting from $3 \mathrm{~h}$ and peaked at $12 \mathrm{~h}$, while p62 levels were greatly attenuated at $3 \mathrm{~h}$ and reached a minimum at $12 \mathrm{~h}$ (Fig. 3C and D). However, p62 protein levels increased markedly at 18 and $24 \mathrm{~h}$ (Fig. 3C and D). These results suggest that PA can induce autophagy in BMSCs at early stages, but autophagy gradually reduced after longer exposure to PA.

PA-induced apoptosis is accelerated by 3-MA and inhibited by $R A$. To investigate the role of autophagy in PA-induced apoptosis of BMSCs, we used 3-MA, a classical inhibitor of autophagy, to inhibit autophagy. Additionally, RA, a classical autophagy inducer, was used to induce autophagy. We measured the viability of PA-treated BMSCs with or without pretreatment with 3-MA or RA. The results showed that BMSCs pretreated with 3-MA for $2 \mathrm{~h}$ had substantially reduced viability. Furthermore, RA pretreatment increased the viability of PA-treated BMSCs (Fig. 4A). In addition, caspase-3/9 activity was evaluated after PA treatment for $24 \mathrm{~h}$, and was found to be increased after pretreatment with 3-MA for $2 \mathrm{~h}$ and decreased after RA pretreatment compared to cells treated with PA alone (Fig. 4B). Moreover, flow cytometry 

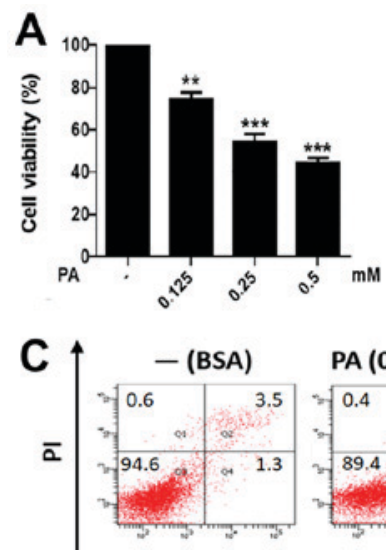

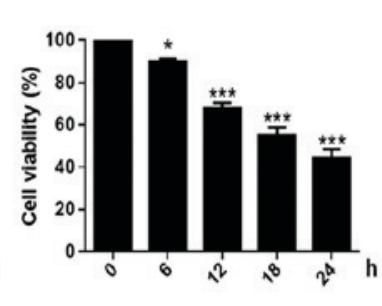

PA (0.125 mM) PA (0.25 mM)

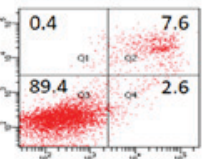

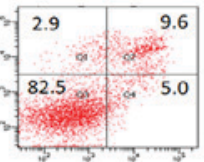

Annexin V-FITC
B
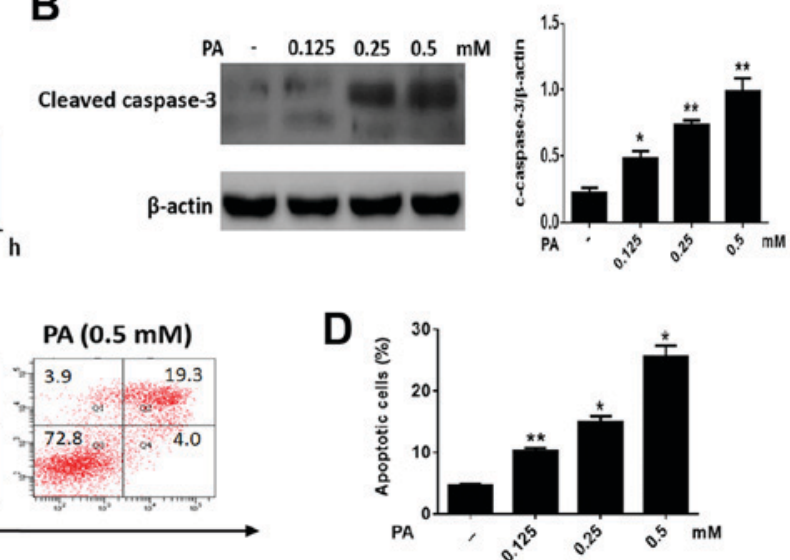

Figure 1. PA promotes apoptosis in BMSCs. (A) Cells were treated with PA $(0.125,0.25$ and 0.5 mM) for 24 h. Cell viability was measured using an MTT assay. The viability of BMSCs treated with PA $(0.5 \mathrm{mM})$ at different times $(0,3,6,12,18$ and $24 \mathrm{~h})$ was detected using an MTT assay. (B) Western blotting was performed to detect the cleaved caspase-3 levels in BMSCs treated with PA $(0.125,0.25$ and $0.5 \mathrm{mM})$ for $24 \mathrm{~h}$. (C) BMSCs treated with PA (0.125, 0.25 and $0.5 \mathrm{mM}$ ) for $24 \mathrm{~h}$ were stained with Annexin V/PI and measured using flow cytometry. (D) The apoptosis ratio of PA-treated BMSCs was obtained by flow cytometry. The apoptotic rate was determined as the percentage of Q2 + Q4. BMSCs treated with BSA were used as the control group. Data are shown as the mean \pm standard error mean from three independent experiments. ${ }^{*} \mathrm{P}<0.05,{ }^{* *} \mathrm{P}<0.01$ and ${ }^{* * * *} \mathrm{P}<0.001 \mathrm{vs}$. control ( 0 mM/0 h). $\mathrm{PA}$, palmitate; BMSCs, bone marrow mesenchymal stem cells; PI, propidium iodide; BSA, bovine serum albumin.

A

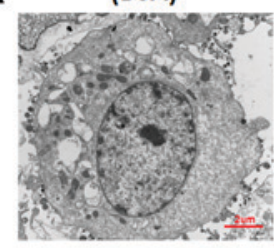

C

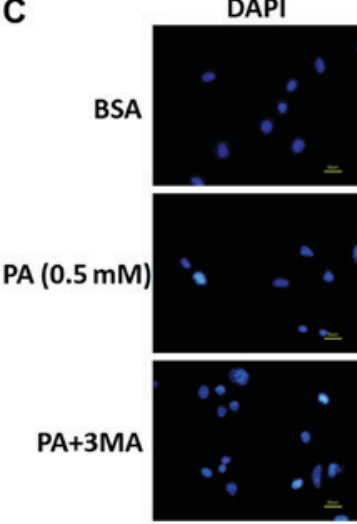

PA (0.5 mM)

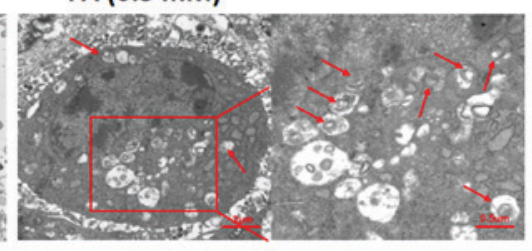

LC3
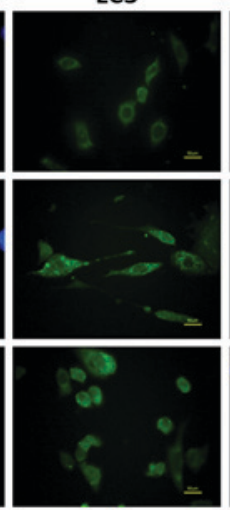

Merge

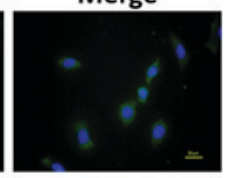

B

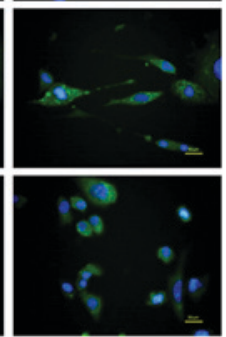

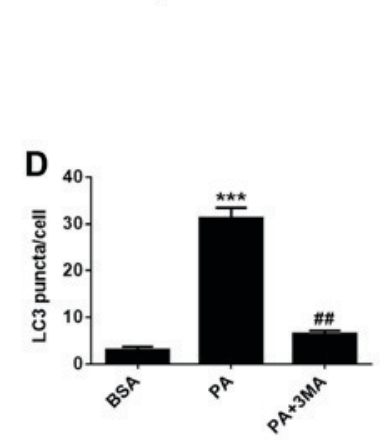

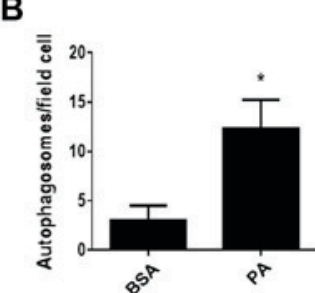

Figure 2. PA induces autophagy in BMSCs. (A) Autophagosomes in BMSCs treated with PA $(0.5 \mathrm{mM})$ for $6 \mathrm{~h}$ were observed by transmission electron microscopy. Arrows indicate autophagosomes. Scale bars, $2 \mu \mathrm{m}$; enlarged areas, $0.5 \mu \mathrm{m}$. (B) Quantitation of autophagosomes. (C) Fluorescence microscopy was used to observe internal LC3 (green) and nuclei (blue) in BMSCs treated with PA (0.5 mM) for $6 \mathrm{~h}$ with or without 3 -MA (5 mM) pretreatment for $2 \mathrm{~h}$. Scale bars, $50 \mu \mathrm{m}$. (D) LC3 puncta were quantified. Data are shown as the mean \pm standard error mean from three independent experiments. * $\mathrm{P}<0.05$ and ${ }^{* * * *} \mathrm{P}<0.001$ vs. BSA; ${ }^{\# \#} \mathrm{P}<0.01$ vs. PA only-treated group. PA, palmitate; BMSCs, bone marrow mesenchymal stem cells; LC3, light chain 3; 3-MA, 3-methyladenine; BSA, bovine serum albumin.

revealed that 3-MA pretreatment increased PA-induced apoptosis (Fig. 4C and D), while RA pretreatment reduced PA-induced apoptosis in BMSCs (Fig. 4E and F).

In addition, western blotting indicated that 3-MA pretreatment decreased the LC3-II levels in BMSCs, and increased cleaved caspase-3 expression levels compared to cells treated with PA alone (Fig. 5A-C). Correspondingly, we found that RA pretreatment increased the levels of LC3-II in BMSCs, and reduced the levels of cleaved caspase-3 (Fig. 5A, C and D).
$P A$ up-regulated ROS production and ROS mediate PA-induced autophagy in BMSCs. After BMSCs were exposed to various concentrations of PA $(0.125,0.25$, $0.50 \mathrm{mM}$ ) for $6 \mathrm{~h}$, intracellular ROS levels were evaluated. Intracellular ROS levels increased as the dose of PA increased (Fig. 6B and C), suggesting that PA up-regulated ROS production in a dose-dependent manner. In addition, NAC, a commonly used antioxidant, was used to inhibit oxidative stress; cells incubated with NAC showed strong inhibition of 
A
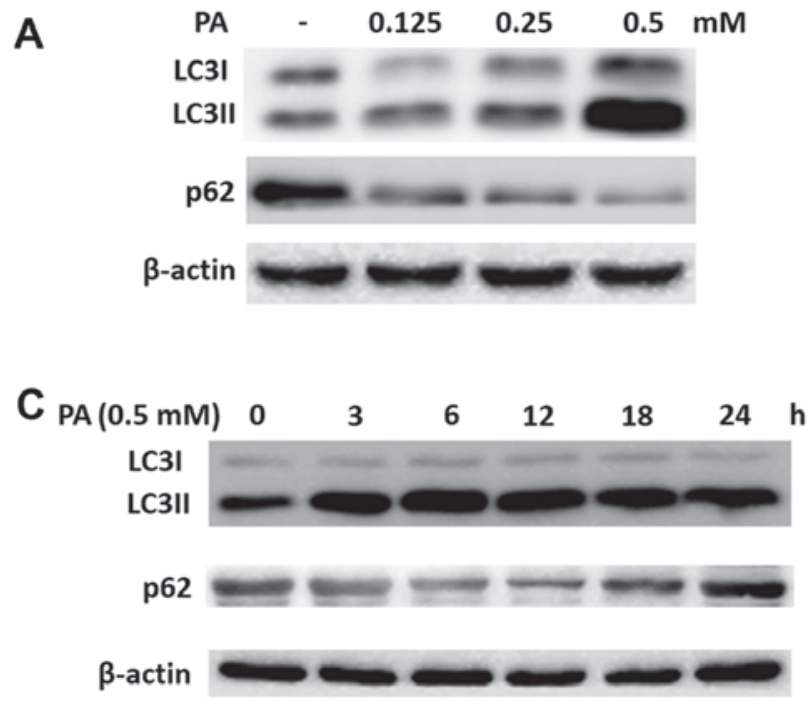
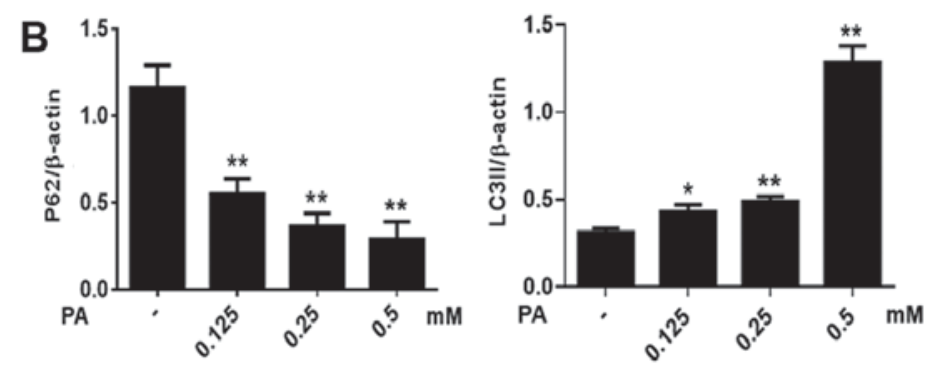

D

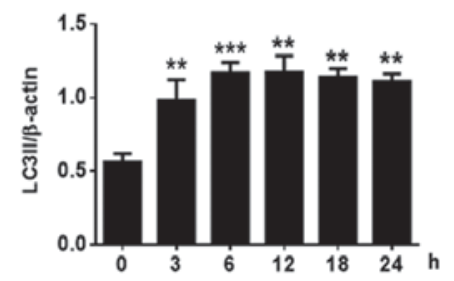

Figure 3. Effect of PA on the expression of LC3II and p62 in BMSCs. (A) Western blotting to detect the expression of LC3 and p62 in BMSCs treated with PA $(0.125,0.25$ and $0.5 \mathrm{mM})$ for $6 \mathrm{~h}$. (B) Quantitative analysis of LC3II and p62 protein expression. (C) The expression of LC3 and p62 in BMSCs treated with PA $(0.5 \mathrm{mM})$ at different times $(0,3,6,12,18$ and $24 \mathrm{~h})$ was detected by western blotting. (D) Quantitative analysis of LC3II and p62 protein expression. Data are shown as the mean \pm standard error mean from three independent experiments. ${ }^{*} \mathrm{P}<0.05,{ }^{* * *} \mathrm{P}<0.01$ and ${ }^{* * * *} \mathrm{P}<0.001$ vs. control $(0 \mathrm{mM} / 0 \mathrm{~h})$; ${ }^{\#} \mathrm{P}<0.05$ and ${ }^{\# \#} \mathrm{P}<0.01$ vs. BMSCs treated with PA for $12 \mathrm{~h}$. PA, palmitate; BMSCs, bone marrow mesenchymal stem cells; LC3, light chain 3.
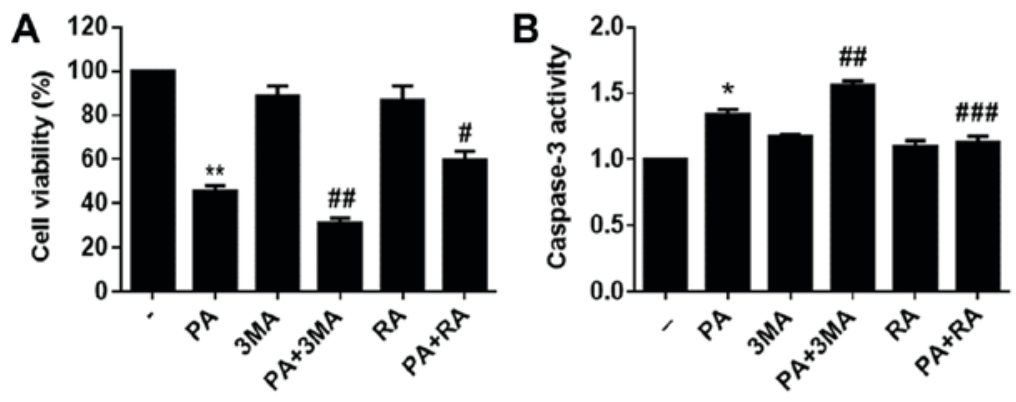

C

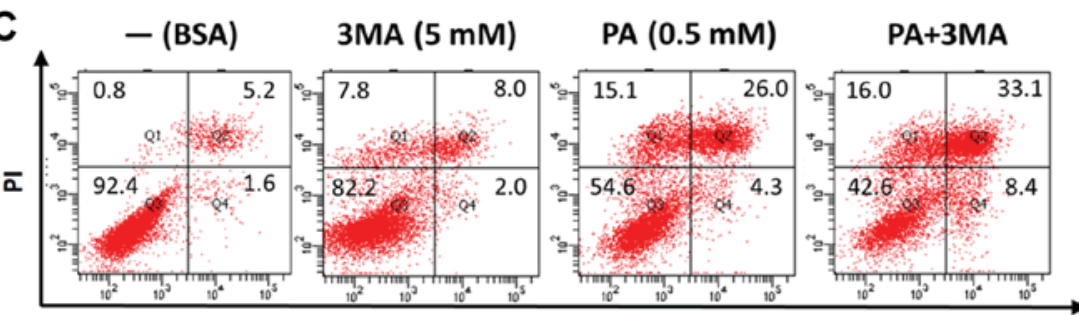

Annexin V-FITC

E

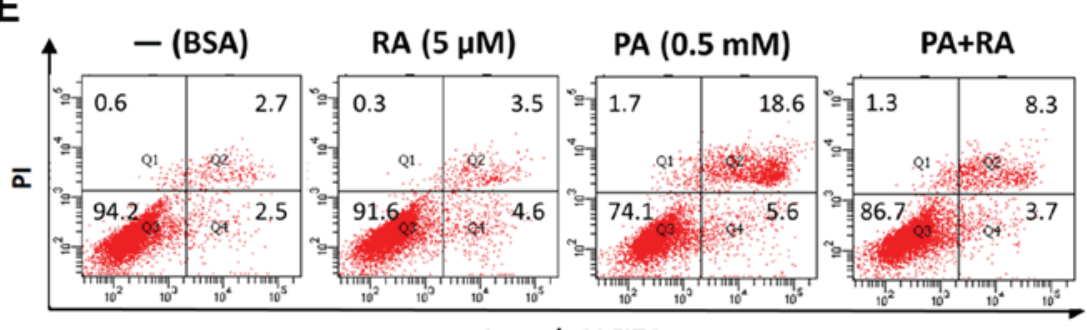

Annexin V-FITC

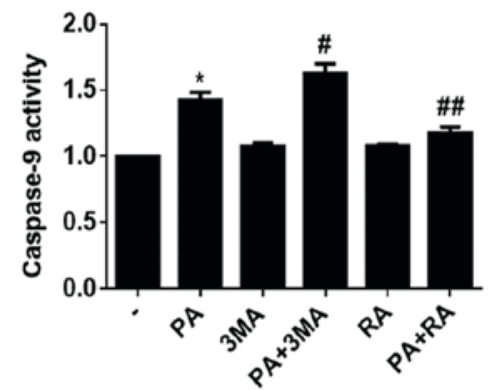

D

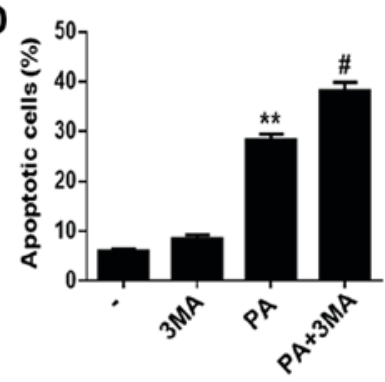

F

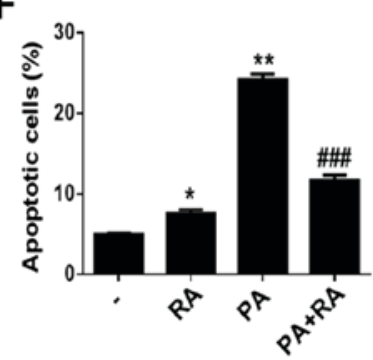

Figure 4. Autophagy protects BMSCs against PA-induced apoptosis. (A) Viability of BMSCs treated with PA (0.5 mM) for 24 h with or without 3-MA $(5 \mathrm{mM})$ or RA $(5 \mu \mathrm{M})$ pretreatment was detected using an MTT assay. (B) Caspase-3/9 activity of BMSCs treated with PA (0.5 mM) for 24 h with or without 3-MA $(5 \mathrm{mM})$ or RA $(5 \mu \mathrm{M})$ pretreatment was evaluated by caspase colorimetric assay kits. (C) Apoptosis of BMSCs treated with PA (0.5 mM) or PA $(0.5 \mathrm{mM})+3$-MA $(5 \mathrm{mM})$ for $24 \mathrm{~h}$ was estimated using Annexin V/PI staining. (D) Quantification analysis of apoptotic cells in 3-MA studies. (E) Apoptosis of BMSCs treated with PA $(0.5 \mathrm{mM})$ or PA $(0.5 \mathrm{mM})+\mathrm{RA}(5 \mu \mathrm{M})$ for $24 \mathrm{~h}$ was estimated using Annexin V/PI staining. (F) Quantification analysis of apoptotic cells in RA studies. The apoptotic rate was determined as the percentage of Q2 + Q4. Data are shown as the mean \pm standard error mean from three independent experiments. ${ }^{*} \mathrm{P}<0.05$ and ${ }^{* *} \mathrm{P}<0.01$ vs. control group; ${ }^{\#} \mathrm{P}<0.05,{ }^{\# \#} \mathrm{P}<0.01$ and ${ }^{\# \# \#} \mathrm{P}<0.001$ vs. PA only-treated group. PA, palmitate; BMSCs, bone marrow mesenchymal stem cells; 3-MA, 3-methyladenine; RA, rapamycin; BSA, bovine serum albumin. 

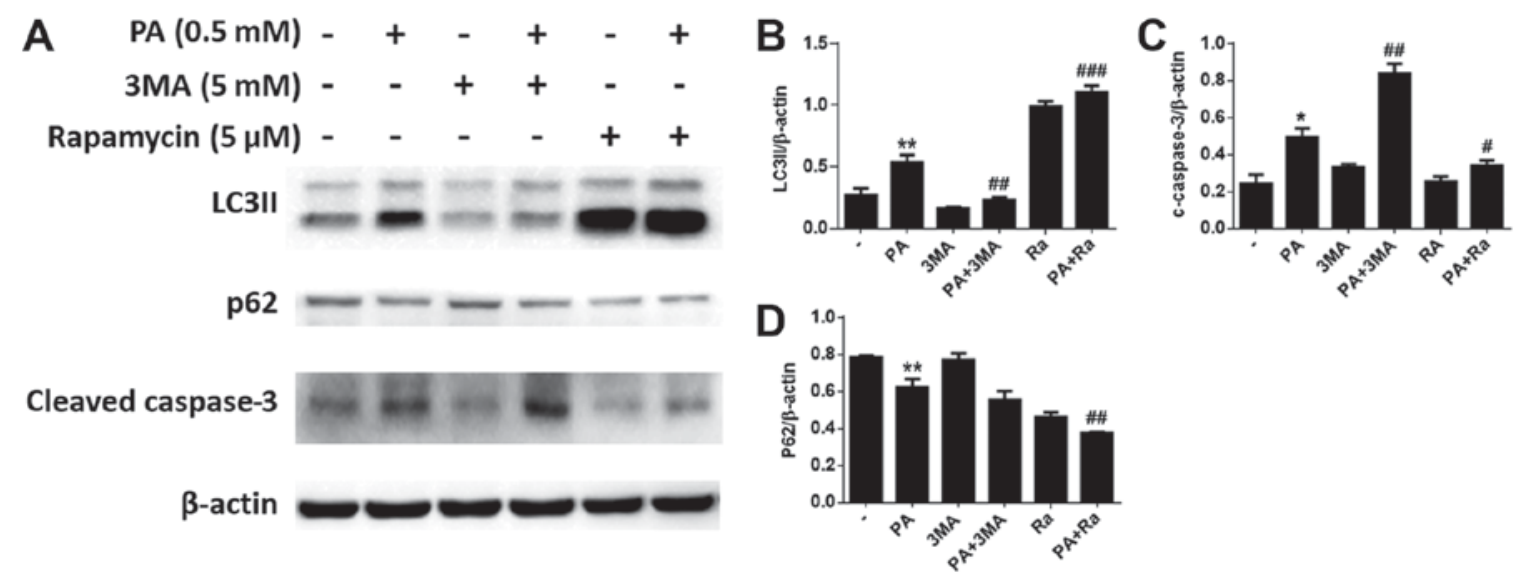

Figure 5. Autophagy protects BMSCs against PA-induced apoptosis. (A) Western blotting to detect the LC3, p62 and cleaved caspase-3 levels in BMSCs treated with PA $(0.5 \mathrm{mM})$ for $24 \mathrm{~h}$ with or without 3-MA $(5 \mathrm{mM})$ or RA $(5 \mu \mathrm{M})$ pretreatment. (B) Quantitative analysis of LC3II protein expression. (C) Quantitative analysis of cleaved caspase-3 protein expression. (D) Quantitative analysis of p62 protein expression. Data are shown as the mean \pm standard error mean from three independent experiments. ${ }^{*} \mathrm{P}<0.05$ and ${ }^{* *} \mathrm{P}<0.01$ vs. control group; ${ }^{\#} \mathrm{P}<0.05,{ }^{\# \#} \mathrm{P}<0.01$ and ${ }^{\# \# \#} \mathrm{P}<0.001$ vs. PA only-treated group. PA, palmitate; BMSCs, bone marrow mesenchymal stem cells; LC3, light chain 3; 3-MA, 3-methyladenine; RA, rapamycin.

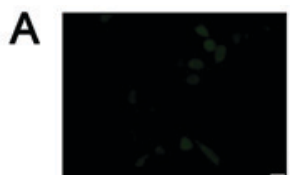

BSA

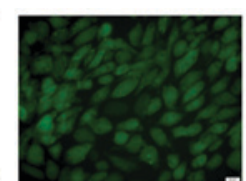

PA (0.5 mM)

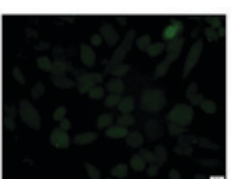

$\mathrm{PA}+\mathrm{NAC}$

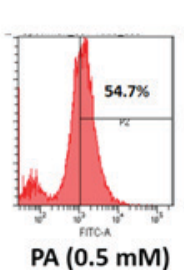

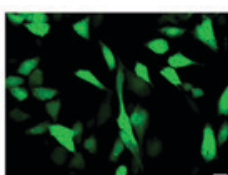

$\mathrm{H}_{2} \mathrm{O}_{2}$

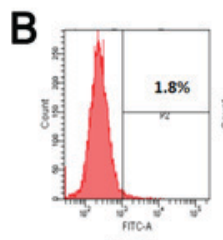

BSA

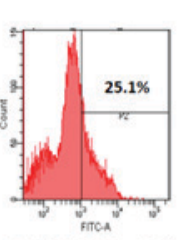

PA $(0.125 \mathrm{mM})$

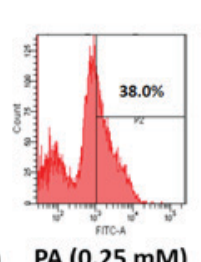

PA (0.25 mM)
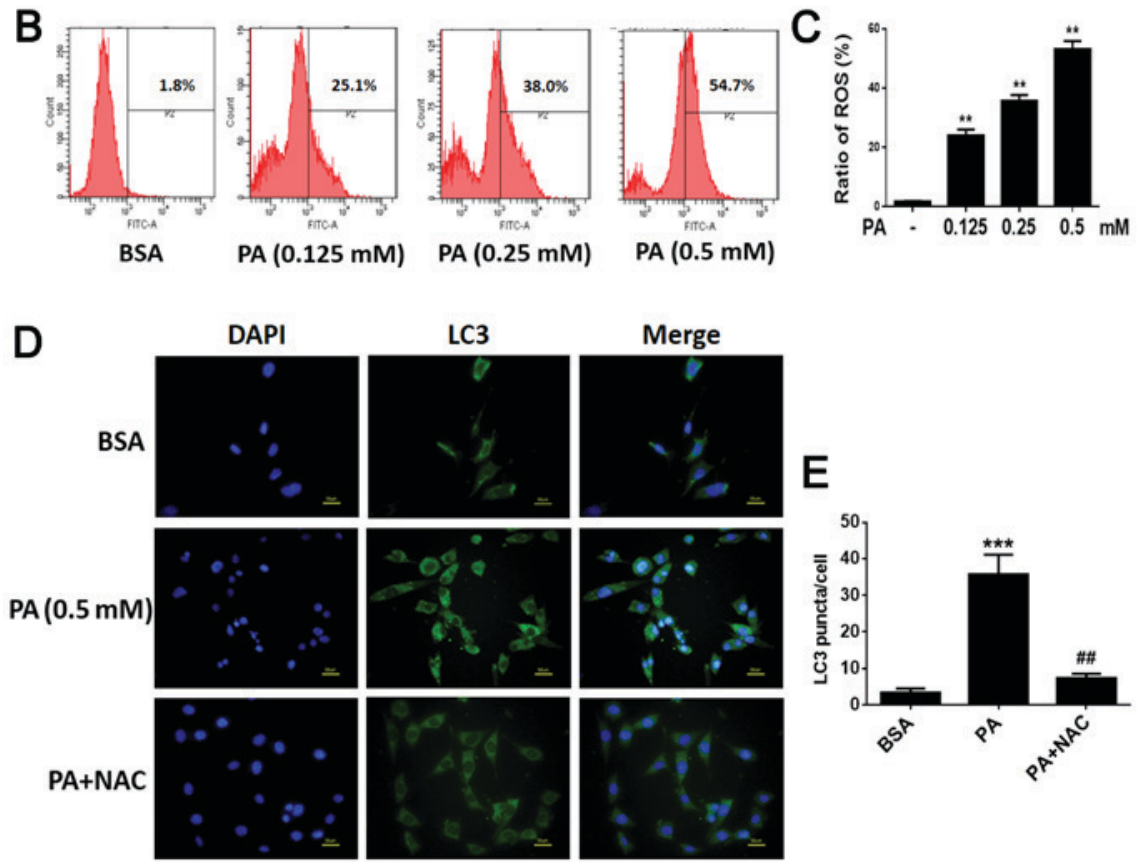

$E$

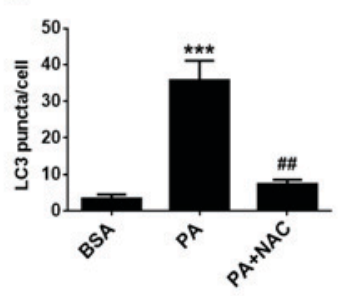

Figure 6. PA upregulates ROS production and in turn, ROS mediates PA-induced autophagy in BMSCs. (A) BMSCs were treated with PA (0.5 mM) for $6 \mathrm{~h}$ with or without NAC (1 mM) and incubated with 2',7'-dichlorodihydrofluorescein diacetate. Fluorescence microscopy was used to measure intracellular ROS levels (magnification, x400). Scale bars, $20 \mu \mathrm{m}$. (B) Following BMSC exposure to PA $(0.125,0.25$ and $0.50 \mathrm{mM})$ for $6 \mathrm{~h}$, intracellular ROS levels were determined. Intracellular ROS levels increased as the dose of PA increased. (C) Quantification analysis of ROS. (D) Fluorescence microscopy was used to observe internal LC3 (green) and nuclei (blue) in BMSCs treated with PA $(0.5 \mathrm{mM})$ for $6 \mathrm{~h}$ with or without NAC (1 mM). Scale bars, $50 \mu \mathrm{m}$. (E) LC3 puncta were quantified. Data are shown as the mean \pm standard error mean from three independent experiments. ${ }^{* *} \mathrm{P}<0.01$ and ${ }^{* * *} \mathrm{P}<0.001 \mathrm{vs}$. control group $(0 \mathrm{mM} / \mathrm{BSA}) ;{ }^{\# \#} \mathrm{P}<0.01$ vs. PA only-treated group. PA, palmitate; ROS, reactive oxygen species; BMSCs, bone marrow mesenchymal stem cells; NAC, N-Acetyl-cysteine; LC3, light chain $3 ; \mathrm{H}_{2} \mathrm{O}_{2}$, hydrogen peroxide.

PA-induced ROS production (Fig. 6A). To explore whether ROS play a role in the activation of PA-induced autophagy, we investigated the role of NAC in autophagy. Western blot analysis showed that NAC markedly decreased the levels of
LC3B-II (Fig. 7A and B). Furthermore, immunofluorescence staining revealed that the rate of LC3-positive cells was lower in cells treated with NAC and PA than in those treated with PA alone (Fig. 6D and E). These results suggest that 
A

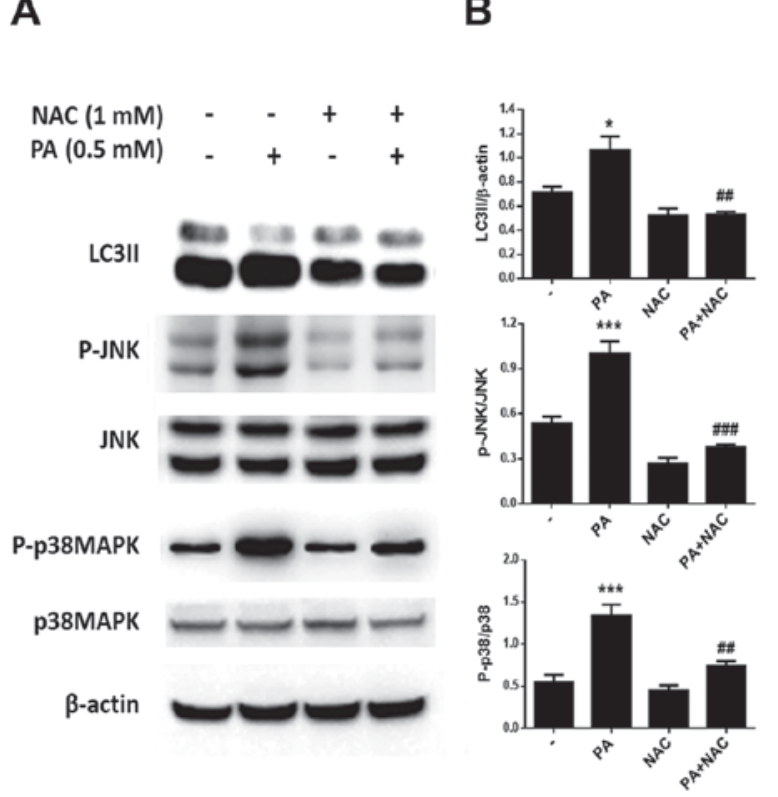

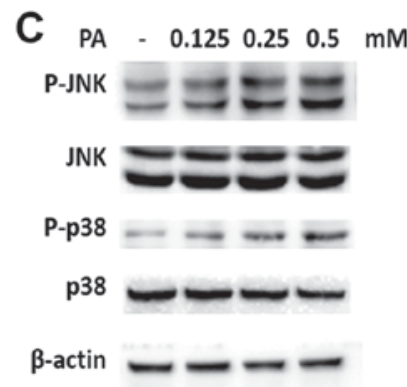

$D^{2}$
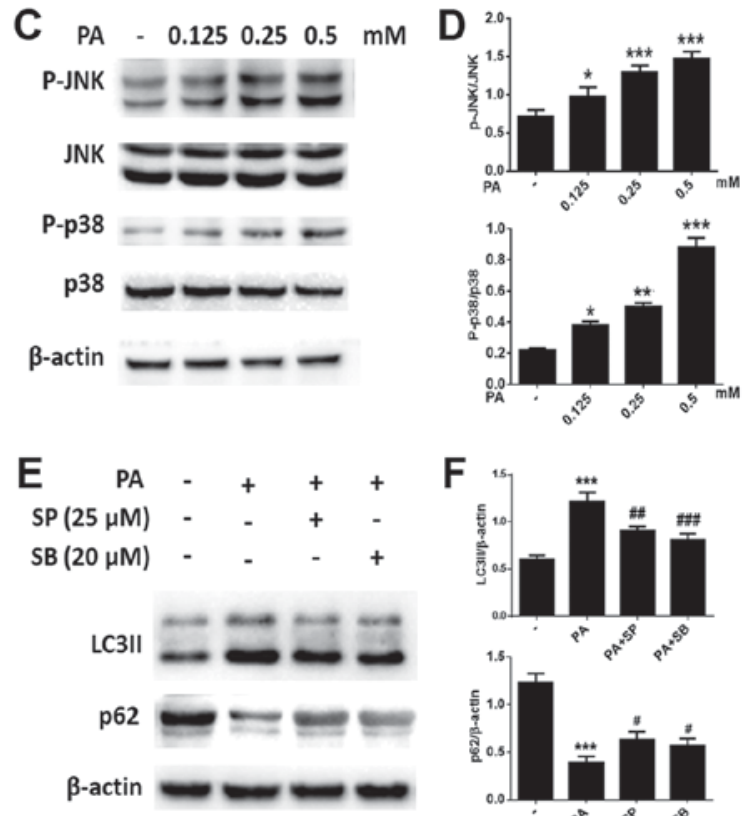

$F$

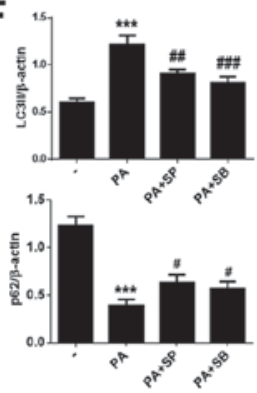

Figure 7. PA induces autophagy through the ROS-JNK/p38 MAPK signaling pathway in BMSCs. (A) Western blotting was used to detect (B) LC3, p-JNK, JNK, p-p38 MAPK and p38 MAPK levels in BMSCs treated with PA $(0.5 \mathrm{mM})$ for $6 \mathrm{~h}$ with or without NAC $(1 \mathrm{mM})$. (C) Western blotting was also performed to determine (D) the levels of p-JNK, JNK, p-p38 MAPK and p38 MAPK in BMSCs treated with PA $(0.125,0.25$ and $0.5 \mathrm{mM})$ for $6 \mathrm{~h}$. (E and F) BMSCs were pretreated with the inhibitor of JNK (SP600125), or the inhibitor of p38 MAPK (SB203580) for $1 \mathrm{~h}$, then treated with PA (0.5 mM) for $6 \mathrm{~h}$. (E) Western blot to detect (F) LC3II and p62 levels in different groups. Data are shown as the mean \pm standard error mean from three independent experiments. "P $<0.05$, ${ }^{* *} \mathrm{P}<0.01$ and ${ }^{* * * *} \mathrm{P}<0.001$ vs. control group; ${ }^{\#} \mathrm{P}<0.05,{ }^{\# \#} \mathrm{P}<0.01$ and ${ }^{\# \# \#} \mathrm{P}<0.001$ vs. PA only-treated group. PA, palmitate; ROS, reactive oxygen species; JNK, c-Jun N-terminal kinase; MAPK, mitogen-activated protein kinase; BMSCs, bone marrow mesenchymal stem cells; LC3, light chain 3; p-, phospho-; NAC, N-Acetyl-cysteine.

PA may induce autophagy by up-regulating ROS levels in BMSCs.

PA induces autophagy through the ROS-JNK/p38 MAPK pathway in BMSCs. We further investigated the mechanism of PA-induced autophagy in BMSCs. Western blotting revealed that the expression of P-JNK and P-p38 MAPK increased with increasing PA concentrations (Fig. 7C and D). Furthermore, pretreatment with the JNK inhibitor SP600125 or the p38 MAPK inhibitor SB203580 greatly reduced PA-induced autophagy (Fig. 7E and F). Furthermore, NAC was used to observe whether ROS is involved in the activation of JNK and p38 MAPK. As expected, the phosphorylation of JNK and p38 MAPK were inhibited by NAC (Fig. 7A and B). Therefore, PA may induce autophagy in BMSCs via the ROS-JNK/p38 MAPK pathway.

\section{Discussion}

The inverse relationship between bone marrow adipose tissue (MAT) and bone mass in the process of aging and the development of osteoporosis has been well established $(24,25)$. Recent studies demonstrated that bone mass and MAT are inversely correlated $(26,27)$. Adipocytes are an important part of the bone marrow, but their physiological function in the bone marrow stem cell niche and the consequence of their excessive accumulation in pathological entities remain unclear (28). Although they have long been considered as inert cells, it is now widely accepted that adipocytes constitute a metabolically active depot that may affect skeletal metabolism (3,29-31). Through the release of FAs, marrow adipocytes may generate a local microenvironment that could impair the viability and function of bone cells (31-33). Recent studies demonstrated that adipocytes liberate factors that inhibit preosteoblast proliferation and differentiation $(34,35)$. Moreover, MSC-derived adipocytes enhanced $\mathrm{Ob}$ apoptosis via the release of saturated FA $(5,36)$. In fact, a higher consumption of saturated FA is negatively correlated with bone mineral density and is associated with an increased risk of osteoporotic fractures $(6,37)$.

As multipotent cells that can differentiate into osteoblasts and chondrocytes, BMSCs are crucial for maintaining bone mass. In osteoporotic and aging patients, the quantity of MSCs is reduced and is correlated with increased MSC apoptosis (38). PA is a highly prevalent saturated FA that can induce apoptosis and autophagy $(20,22)$. We found that PA-treated BMSCs showed a considerable reduction in cell viability in a time- and dose-dependent manner. This result was validated by increased expression of the apoptotic factor cleaved caspase-3. The Annexin V/PI staining assay further confirmed the apoptotic effects of PA.

Both autophagy and apoptosis have been identified as key determinants in skeletal maintenance $(13,39)$. PA-induced autophagy is considered to be a ubiquitous process, and has been induced in other cell lines (40). When autophagy is induced, LC3 levels are up-regulated. In this study, western blotting revealed that the levels of LC3-II increased greatly after incubation for 3-12 h. p62, a biomarker of the degradation of autolysosomes that is negatively correlated with autophagy, was decreased in BMSCs treated with PA from 3-12 h; this 
is in accordance with the change in LC3 levels, implying the activation of autophagy. However, after $12 \mathrm{~h}$, p62 began to accumulate, p62 is a substrate and regulatory protein of mammalian autophagy (41). The cellular p62 level is inversely proportional to the number of autophagy lysosomes and is an important indicator of autophagosome clearance. The increase of p62 observed here suggested that the autophagy/ lysosomal degradation pathway was inhibited, and autophagic flow was blocked and gradually weakened, suggesting that autophagy was gradually weakened after longer exposure to PA. Consistent with the changes of $\mathrm{p} 62$ protein levels, previous research found that, in $\mathrm{H} 9 \mathrm{c} 2$ cells, the phosphorylation of p70S6K, a direct downstream target of mTORC1, was reduced from 6 to $18 \mathrm{~h}$, and then increased thereafter, following PA treatment for $24 \mathrm{~h}$, suggesting that the activity of mTORC1 was initially suppressed when PA promoted autophagic flux in the case of short-term exposure (42). However, mTORC1 was reactivated and the autophagic flux was blocked by prolonged PA treatment, thus leading to the decrease of autophagy.

Studies have suggested that autophagy could inhibit apoptosis and facilitate cell survival $(20,43)$. A recent study suggested that autophagy induction could lead to a survival response against oxidative stress in BMSCs (44). We found that further increases in autophagy induced by RA reversed PA-induced apoptosis. Conversely, the proportion of apoptotic cells was increased when autophagy was inhibited by 3-MA. This finding was consistent with previous results showing that autophagy may play a protective role in BMSCs to prevent PA-induced apoptosis. Taken together, these results suggest that further induction of autophagy could reverse PA-induced apoptosis in BMSCs at early stages. However, as autophagy gradually weakened after $12 \mathrm{~h}$, its protective effect against PA-induced apoptosis was limited. Since apoptosis is inevitable under prolonged PA exposure, the number of apoptotic BMSCs increased significantly.

Under normal physiological conditions, the generation of ROS is a regular part of cellular metabolism (45). However, research on the lipotoxicity of adipocytes has demonstrated that excessive and long-term treatment with PA induced excessive accumulation of intracellular ROS in different cell types (46-49). It is believed that ROS are produced via multiple processes, such as the mitochondrial electron transport chain, NOX, nitric-oxide synthase, and xanthine oxidase (46). Among these possibilities, NOX may be the predominant source of ROS in different cell types $(46,47,49)$. Previous studies have shown that PA exposure causes Src activation and increased NOX activity through Src signaling, leading to overproduction of ROS (46). It is well known that excessive levels of ROS not only directly damage cells by oxidizing DNA, protein, and lipid, but also indirectly damage cells by activating a variety of stress-sensitive intracellular signaling pathways $(50,51)$. Meanwhile, accumulation of ROS and reactive nitrogen species cause oxidative stress, which has been proposed to induce autophagy in different cell types $(42,44)$. In this study, we found that PA up-regulated ROS production in a dose-dependent manner in BMSCs. In addition, pretreating cells with NAC, a well-known antioxidant, strongly inhibited PA-induced autophagy in BMSCs. These results suggested that PA induced accumulation of intracellular ROS in BMSCs. Furthermore, excessive ROS accumulation might disturb cellular homeostasis, resulting in apoptosis (52). Meanwhile, as a protective mechanism under various environmental stresses, autophagy is also activated to decrease the rate of ROS-induced apoptosis.

Previous studies have suggested that apoptosis and autophagy might be triggered by common upstream signals. On a molecular level, this means that the apoptotic and autophagic response mechanisms share common pathways $(53,54)$. ROS production may lead to oxidative stress that mediates increased autophagy and decreased ROS-induced apoptosis (55). In addition, apoptotic stimuli may trigger JNK and p38 MAPK activation through ROS production $(18,56)$. ROS has also been shown to be closely related to this signal pathway $(57,58)$. A recent study suggested that autophagy was induced in colon cancer cells treated with bufalin, an anti-cancer drug. Bufalin relies on ROS generation, which leads to the activation of the JNK pathway (59). In our study, we found that PA promoted the activation of JNK and p38 MAPK in BMSCs in a dose-dependent manner. We also investigated whether this signal pathway was involved in PA-induced autophagy. Pretreatment with the JNK inhibitor SP600125 or the p38 MAPK inhibitor SB203580 markedly reduced PA-induced autophagy and inhibited PA-triggered activation of JNK and p38 MAPK. Taken together, these results indicate that the ROS-JNK/p38 MAPK pathway plays an important role in PA-induced autophagy.

In conclusion, the present study demonstrated that PA can induce autophagy, and that prolonged PA treatment may reduce autophagy in BMSCs. Moreover, we found that autophagy can protect cells against PA-induced apoptosis in BMSCs. Furthermore, PA might induce autophagy through the ROS-JNK/p38 MAPK pathway in BMSCs. Combined with the above conclusions and our findings, we postulated that apoptosis induced by palmitate in this in vitro assay may serve as an explanation for osteoporosis in high saturated FA consumption people. Our findings can improve the general understanding of the mechanisms through which BMSCs adapt to PA-induced apoptosis. This study also provides a novel approach for the prevention and treatment of PA-induced lipotoxicity in bone cells. However, it is undeniable that the pathogenesis of osteoporosis is complex, and the relationship between FA and osteoporosis still requires in vivo experiments for further studies.

\section{Acknowledgements}

The authors would like to thank the Molecular Biology Center Laboratory of China Medical University (Shenyang, China).

\section{Funding}

The present study was supported by the Natural Science Foundation of Liaoning Province (grant no. 201102282) and the Education Science Foundation of Liaoning Province (grant no. L2014417).

\section{Availability of data and materials}

The datasets used and/or analyzed during the current study are available from the corresponding author on reasonable request. 


\section{Authors' contributions}

YYL and QWL conceived and designed the experiments. YYL, SKZ and NW performed the experiments. YYL, NW and SKZ conducted the data analysis, and YYL produced the manuscript. All authors have read and approved the final manuscript.

\section{Ethics approval and consent to participate}

Not applicable.

\section{Consent for publication}

Not applicable.

\section{Competing interests}

The authors declare that they have no competing interests.

\section{References}

1. Brown SA and Rosen CJ: Osteoporosis. Med Clin North Am 87: 1039-1063, 2003.

2. Gimble JM, Zvonic S, Floyd ZE, Kassem M and Nuttall ME: Playing with bone and fat. J Cell Biochem 98: 251-266, 2006.

3. Rosen CJ and Bouxsein ML: Mechanisms of disease: Is osteoporosis the obesity of bone? Nat Clin Pract Rheumatol 2: 35-43, 2006.

4. Pei L and Tontonoz P: Fat's loss is bone's gain. J Clin Invest 113: 805-806, 2004

5. Elbaz A, Wu X, Rivas D, Gimble JM and Duque G: Inhibition of fatty acid biosynthesis prevents adipocyte lipotoxicity on human osteoblasts in vitro. J Cell Mol Med 14: 982-991, 2010.

6. Orchard TS, Cauley JA, Frank GC, Neuhouser ML, Robinson JG, Snetselaar L, Tylavsky F, Wactawski-Wende J, Young AM, Lu B and Jackson RD: Fatty acid consumption and risk of fracture in the Women's Health Initiative. Am J Clin Nutr 92: 1452-1460, 2010.

7. Turpin SM, Lancaster GI, Darby I, Febbraio MA and Watt MJ Apoptosis in skeletal muscle myotubes is induced by ceramides and is positively related to insulin resistance. Am J Physiol Endocrinol Metab 291: E1341-E1350, 2006.

8. Malhi H, Bronk SF, Werneburg NW and Gores GJ: Free fatty acids induce JNK-dependent hepatocyte lipoapoptosis. J Biol Chem 281: 12093-12101, 2006.

9. Kim JE, Ahn MW, Baek SH, Lee IK, Kim YW, Kim JY, Dan JM and Park SY: AMPK activator, AICAR, inhibits palmitate-induced apoptosis in osteoblast. Bone 43: 394-404, 2008.

10. de Bruin EC and Medema JP: Apoptosis and non-apoptotic deaths in cancer development and treatment response. Cancer Treat Rev 34: 737-749, 2008.

11. Levine B and Kroemer G: Autophagy in the pathogenesis of disease. Cell 132: 27-42, 2008.

12. Eisenberg-Lerner A, Bialik S, Simon HU and Kimchi A: Life and death partners: Apoptosis, autophagy and the cross-talk between them. Cell Death Differ 16: 966-975, 2009.

13. Hocking LJ, Whitehouse C and Helfrich MH: Autophagy: A new player in skeletal maintenance? J Bone Miner Res 27: 1439-1447, 2012.

14. Caramés B, Taniguchi N, Otsuki S, Blanco FJ and Lotz M: Autophagy is a protective mechanism in normal cartilage, and its aging-related loss is linked with cell death and osteoarthritis. Arthritis Rheum 62: 791-801, 2010.

15. Lu J, Wang Q, Huang L, Dong H, Lin L, Lin N, Zheng F and Tan J: Palmitate causes endoplasmic reticulum stress and apoptosis in human mesenchymal stem cells: Prevention by AMPK activator. Endocrinology 153: 5275-5284, 2012.

16. Gillet C, Spruyt D, Rigutto S, Dalla Valle A, Berlier J, Louis C, Debier C, Gaspard N, Malaisse WJ, Gangji V and Rasschaert J: Oleate abrogates palmitate-induced lipotoxicity and proinflammatory response in human bone marrow-derived mesenchymal stem cells and osteoblastic cells. Endocrinology 156: 4081-4093, 2015.
17. Dong X, Bi L, He S, Meng G, Wei B, Jia S and Liu J: FFAs-ROS-ERK/P38 pathway plays a key role in adipocyte lipotoxicity on osteoblasts in co-culture. Biochimie 101: 123-131, 2014.

18. Li Y, Luo Q, Yuan L, Miao C, Mu X, Xiao W, Li J, Sun T and Ma E: JNK-dependent Atg4 upregulation mediates asperphenamate derivative BBP-induced autophagy in MCF-7 cells. Toxicol Appl Pharmacol 263: 21-31, 2012.

19. Sui X, Kong N, Ye L, Han W, Zhou J, Zhang Q, He C and Pan H: p38 and JNK MAPK pathways control the balance of apoptosis and autophagy in response to chemotherapeutic agents. Cancer Lett 344: 174-179, 2014.

20. Choi SE, Lee SM, Lee YJ, Li LJ, Lee SJ, Lee JH, Kim Y, Jun HS, Lee KW and Kang Y: Protective role of autophagy in palmitate-induced INS-1 beta-cell death. Endocrinology 150: 126-134, 2009.

21. Martino L, Masini M, Novelli M, Beffy P, Bugliani M, Marselli L, Masiello P, Marchetti P and De Tata V: Palmitate activates autophagy in INS-1E $\beta$-cells and in isolated rat and human pancreatic islets. PLoS One 7: e36188, 2012.

22. Xie W, Zhai Z, Yang Y, Kuang T and Wang C: Free fatty acids inhibit TM-EPCR expression through JNK pathway: An implication for the development of the prothrombotic state in metabolic syndrome. J Thromb Thrombolysis 34: 468-474, 2012.

23. Griffith JF, Yeung DK, Ahuja AT, Choy CW, Mei WY, Lam SS, Lam TP, Chen ZY and Leung PC: A study of bone marrow and subcutaneous fatty acid composition in subjects of varying bone mineral density. Bone 44: 1092-1096, 2009.

24. Justesen J, Stenderup K, Ebbesen EN, Mosekilde L, Steiniche T and Kassem M: Adipocyte tissue volume in bone marrow is increased with aging and in patients with osteoporosis. Biogerontology 2: 165-171, 2001.

25. Hardouin P, Pansini V and Cortet B: Bone marrow fat. Joint Bone Spine 81: 313-319, 2014.

26. Wren TA, Chung SA, Dorey FJ, Bluml S, Adams GB and Gilsanz V: Bone marrow fat is inversely related to cortical bone in young and old subjects. J Clin Endocrinol Metab 96: 782-786, 2011.

27. Shen W, Chen J, Gantz M, Punyanitya M, Heymsfield SB, Gallagher D, Albu J, Engelson E, Kotler D, Pi-Sunyer X and Gilsanz V: MRI-measured pelvic bone marrow adipose tissue is inversely related to DXA-measured bone mineral in younger and older adults. Eur J Clin Nutr 66: 983-988, 2012.

28. Fazeli PK, Horowitz MC, MacDougald OA, Scheller EL, Rodeheffer MS, Rosen CJ and Klibanski A: Marrow fat and bone-new perspectives. J Clin Endocrinol Metab 98: 935-945, 2013.

29. Ahima RS and Flier JS: Adipose tissue as an endocrine organ. Trends Endocrinol Metab 11: 327-332, 2000.

30. Frühbeck G, Gómez-Ambrosi J, Muruzábal FJ and Burrell MA: The adipocyte: A model for integration of endocrine and metabolic signaling in energy metabolism regulation. Am J Physiol Endocrinol Metab 280: E827-E847, 2001.

31. Lecka-Czernik B: Marrow fat metabolism is linked to the systemic energy metabolism. Bone 50: 534-539, 2012.

32. Rosen CJ, Ackert-Bicknell C, Rodriguez JP and Pino AM: Marrow fat and the bone microenvironment: Developmental, functional, and pathological implications. Crit Rev Eukaryot Gene Expr 19: 109-124, 2009.

33. Kawai M, de Paula FJ and Rosen CJ: New insights into osteoporosis: The bone-fat connection. J Intern Med 272: 317-329, 2012.

34. Maurin AC, Chavassieux PM, Frappart L, Delmas PD, Serre CM and Meunier PJ: Influence of mature adipocytes on osteoblast proliferation in human primary cocultures. Bone 26: 485-489, 2000.

35. Clabaut A, Delplace S, Chauveau C, Hardouin P and Broux O: Human osteoblasts derived from mesenchymal stem cells express adipogenic markers upon coculture with bone marrow adipocytes. Differentiation 80: 40-45, 2010.

36. Wang D, Haile A and Jones LC: Dexamethasone-induced lipolysis increases the adverse effect of adipocytes on osteoblasts using cells derived from human mesenchymal stem cells. Bone 53: 520-530, 2013.

37. Corwin RL, Hartman TJ, Maczuga SA and Graubard BI: Dietary saturated fat intake is inversely associated with bone density in humans: Analysis of NHANES III. J Nutr 136: 159-165, 2006.

38. Fibbe WE and Noort WA: Mesenchymal stem cells and hematopoietic stem cell transplantation. Ann N Y Acad Sci 996: 235-244, 2003

39. Manolagas SC and Parfitt AM: What old means to bone. Trends Endocrinol Metab 21: 369-374, 2010. 
40. Komiya K, Uchida T, Ueno T, Koike M, Abe H, Hirose T, Kawamori R, Uchiyama Y, Kominami E, Fujitani Y and Watada H: Free fatty acids stimulate autophagy in pancreatic $\beta$-cells via JNK pathway. Biochem Biophys Res Commun 401: 561-567, 2010.

41. Bjørkøy G,Lamark T, Brech A, Outzen H,Perander M, Overvatn A, Stenmark H and Johansen T: p62/SQSTM1 forms protein aggregates degraded by autophagy and has a protective effect on huntingtin-induced cell death. J Cell Biol 171: 603-614, 2005.

42. Liu J, Chang F, Li F, Fu H, Wang J, Zhang S, Zhao J and Yin D: Palmitate promotes autophagy and apoptosis through ROS-dependent JNK and p38 MAPK. Biochem Biophys Res Commun 463: 262-267, 2015.

43. Cai N, Zhao X, Jing Y, Sun K, Jiao S, Chen X, Yang H, Zhou Y and Wei L: Autophagy protects against palmitate-induced apoptosis in hepatocytes. Cell Biosci 4: 28, 2014.

44. Song C, Song C and Tong F: Autophagy induction is a survival response against oxidative stress in bone marrow-derived mesenchymal stromal cells. Cytotherapy 16: 1361-1370, 2014.

45. Kiffin R, Bandyopadhyay U and Cuervo AM: Oxidative stress and autophagy. Antioxid Redox Signal 8: 152-162, 2006.

46. Gao D, Nong S, Huang X, Lu Y, Zhao H, Lin Y, Man Y, Wang S, Yang $\mathbf{J}$ and $\mathrm{Li} \mathrm{J}$ : The effects of palmitate on hepatic insulin resistance are mediated by NADPH Oxidase 3-derived reactive oxygen species through JNK and p38MAPK pathways. J Biol Chem 285: 29965-29973, 2010.

47. Sato Y, Fujimoto S, Mukai E, Sato H, Tahara Y, Ogura K, Yamano G, Ogura M, Nagashima K and Inagaki N: Palmitate induces reactive oxygen species production and $\beta$-cell dysfunction by activating nicotinamide adenine dinucleotide phosphate oxidase through Src signaling. J Diabetes Investig 5: 19-26, 2014.

48. Wei CD, Li Y,Zheng HY, Tong YQ and Dai W: Palmitate induces $\mathrm{H} 9 \mathrm{c} 2$ cell apoptosis by increasing reactive oxygen species generation and activation of the ERK1/2 signaling pathway. Mol Med Rep 7: 855-861, 2013.

49. Zhang M, Wang CM, Li J, Meng ZJ, Wei SN, Li J, Bucala R, Li YL and Chen L: Berberine protects against palmitate-induced endothelial dysfunction: Involvements of upregulation of AMPK and eNOS and downregulation of NOX4. Mediators Inflamm 2013: 260464, 2013.
50. Klaunig JE, Kamendulis LM and Hocevar BA: Oxidative stress and oxidative damage in carcinogenesis. Toxicol Pathol 38: 96-109, 2010.

51. Ghosh J, Das J, Manna P and Sil PC: Taurine prevents arsenic-induced cardiac oxidative stress and apoptotic damage: Role of NF-kappa B, p38 and JNK MAPK pathway. Toxicol Appl Pharmacol 240: 73-87, 2009.

52. Manolagas SC: From estrogen-centric to aging and oxidative stress: A revised perspective of the pathogenesis of osteoporosis. Endocr Rev 31: 266-300, 2010.

53. Maiuri MC, Zalckvar E, Kimchi A and Kroemer G: Self-eating and self-killing: Crosstalk between autophagy and apoptosis. Nat Rev Mol Cell Biol 8: 741-752, 2007.

54. Zhang YH, Wu YL, Tashiro S, Onodera S and Ikejima T: Reactive oxygen species contribute to oridonin-induced apoptosis and autophagy in human cervical carcinoma HeLa cells. Acta Pharmacol Sin 32: 1266-1275, 2011.

55. Lv XC and Zhou HY: Resveratrol protects $\mathrm{H} 9 \mathrm{c} 2$ embryonic rat heart derived cells from oxidative stress by inducing autophagy: role of p38 mitogen-activated protein kinase. Can J Physiol Pharm 90: 655-662, 2012.

56. Dolado I, Swat A, Ajenjo N, De Vita G, Cuadrado A and Nebreda AR: p38alpha MAP kinase as a sensor of reactive oxygen species in tumorigenesis. Cancer Cell 11: 191-205, 2007.

57. West RJ and Sweeney ST: Oxidative stress and autophagy: Mediators of synapse growth? Autophagy 8: 284-285, 2012.

58. Haberzettl P and Hill BG: Oxidized lipids activate autophagy in a JNK-dependent manner by stimulating the endoplasmic reticulum stress response. Redox Biol 1: 56-64, 2013.

59. Xie CM, Chan WY, Yu S, Zhao J and Cheng CH: Bufalin induces autophagy-mediated cell death in human colon cancer cells through reactive oxygen species generation and JNK activation. Free Radic Biol Med 51: 1365-1375, 2011. 